\title{
Estimation of wave runup on shorelines based on long-term variation of wave conditions
}

\author{
Dag Myrhaug
}

Received: 13 August 2014 / Accepted: 5 February 2015 / Published online: 13 March 2015

(C) Springer International Publishing AG 2015

\begin{abstract}
This article provides a simple analytical method which can be used to give estimates of the wave runup on shorelines based on long-term variation of wave conditions. This is achieved by providing bivariate distribution of significant wave height with the wave runup. This wave runup is defined in terms of significant wave height and spectral peak period in deep water. This article presents the mean value and the standard deviation, that is, more precisely the conditional expected value and the conditional variance of the wave runup for given significant wave height. Examples of results corresponding to typical field conditions are also given. Based on, for example, global wave statistics, the present analytical results can be used to make estimates of the wave runup.
\end{abstract}

Keywords Wave runup - Significant wave height . Bivariate distributions - Coastal protection work

\section{Introduction}

The present work addresses random wave runup on beaches and coastal structures such as breakwaters, seawalls and artificial reefs. For beaches and such structures it is essential to make reliable assessments of the maximum wave runup to be able to take necessary protection measures. The recent focus on extreme phenomena induced by climate change has generated new interest in the analysis of wave runup on shorelines [see, e.g., de la Pena et al. (2014)].

D. Myrhaug ( $)$

Department of Marine Technology, Norwegian University of Science and Technology, 7491 Trondheim, Norway

e-mail: dag.myrhaug@ntnu.no
The wave runup height is defined as the vertical difference between the highest point of wave runup and the still water level. Here, the runup is given by two components; the wave setup and the swash. The wave setup is the mean water elevation level (e.g., referring to the deep water level) and is caused by the radiation stress [see, e.g., Dean and Dalrymple (1984)]. The swash motion oscillates from the wave setup and is taken as the interception between the sea and the beach or structure [see de la Pena et al. (2014) for more details]. Due to the stochastic nature of waves, many of the commonly used design formulas use the runup height $\left(R_{2}\right)$, which is the runup height exceeded by $2 \%$ of the runup maxima at the toe of the beach or structure. de la Pena et al. (2014) presented a new formulation for the wave runup $\left(R_{2}\right)$ on a shoreline in terms of the significant wave height $\left(H_{S}\right)$ in deep water, the slope of the beach, and the surf parameter defined in terms of $H_{s}$ and the spectral peak period $\left(T_{p}\right)$ in deep water. de la Pena et al. (2014) included also a literature review as well as a summary of previous wave runup formulations.

The purpose of this study is to present a simple analytical method which can be used to give estimates of the wave runup $R_{2}$ based on long-term variation of wave conditions of $\left(H_{s}, T_{p}\right)$ or $\left(H_{s}, T_{z}\right)$, where $T_{z}$ is the mean zero-crossing wave period. This is obtained by providing parametric models of joint distributions of $\left(H_{s}, R_{2}\right)$. This is achieved by adopting de la Pena et al.'s (2014) runup formulation and by transforming the parametric models of the joint $\left(H_{s}, T_{p}\right)$ and $\left(H_{S}, T_{z}\right)$ distributions fitted to field data by Moan et al. (2005) and Bitner-Gregersen and Guedes Soares (2007), respectively. Examples of calculating the conditional mean values and conditional standard deviations of $R_{2}$ given $H_{S}$ corresponding to typical field conditions are also provided to demonstrate the application of the method. Thus, it is demonstrated how joint $\left(H_{s}, T_{p}\right)$ or $\left(H_{s}, T_{z}\right)$ distributions can be used to provide estimates of wave runup, and how, 
for example, global wave statistics can be used to calculate this.

The long-term variation of wave conditions referred to in this paper, and as presented in Moan et al. (2005) and BitnerGregersen and Guedes Soares (2007), is expressed in terms of $H_{S}, T_{p}$ (or $T_{z}$ ) assuming stationary sea state conditions with a duration of, e.g., $3 \mathrm{~h}$ based on a 20 min time series of the free surface elevation. The number of occurrences of each sea state is described by a frequency table or scatter diagram representing a discrete (long-term) joint $H_{s}, T_{p}$ (or $T_{z}$ ) distribution of the wave conditions at the location considered.

\section{Background}

Several laboratory and field experiments have been performed to study extreme runup events, and equations for the estimation of the $2 \%$ exceedance value of runup maxima, $R_{2}$, have been provided. de la Pena et al. (2014) have recently given a review of various formulations (see their Table 1), which can be represented by

$\frac{R_{2}}{H_{S}}=K \xi_{p}$

where $K$ is a coefficient, and $H_{s}$ is the significant wave height in deep water (i.e., representing one storm condition with a duration of, e.g., $3 \mathrm{~h}$ and with a return period specified by the user). Moreover, $\xi_{p}$ is the surf parameter defined as

$\xi_{p}=m\left(\frac{H_{s}}{\frac{g}{2 \pi} T_{p}^{2}}\right)^{-1 / 2}$

where $m=\tan \alpha$ is the slope with an angle $\alpha$ with the horizontal, $T_{p}$ is the spectral peak period, and $g$ is the acceleration due to gravity. Thus, this surf parameter is defined in terms of sea state parameters in deep water. Substitution of Eq. (2) in (1) can be rearranged to give (by defining $R$ as a mathematical help)

$R \equiv \frac{R_{2}}{K m \sqrt{g / 2 \pi}}=T_{p} \sqrt{H_{s}}$

If, e.g., the mean zero-crossing wave period is given, then relationships between $T_{p}$ and $T_{z}$ exist, for example, given by

Table 1 Weibull parameters for $H_{s}$, see Eq. (7)

\begin{tabular}{llll}
\hline BGGS07 & $s$ & $r$ & $t$ \\
\hline Dataset 1 & 3.104 & 1.357 & 0.906 \\
Dataset 2 & 2.848 & 1.419 & 1.021 \\
Dataset 3 & 2.939 & 1.240 & 0.896 \\
Dataset 4 & 2.857 & 1.449 & 0.838 \\
Dataset 5 & 2.420 & 1.169 & 1.258 \\
\hline
\end{tabular}

$T_{p}=c T_{z}$ where $c$ is a coefficient. Here, Eq. (3) is rearranged to

$R=\frac{R_{2}}{c K m \sqrt{g / 2 \pi}}=T \sqrt{H_{S}}$

where $T$ represents $T_{p}$ or $T_{z}$. Thus, $R$ (and $R_{2}$ ) is defined in terms of the sea state parameters $H_{s}$ and $T$ (i.e., $T_{p}$ or $T_{z}$ ) in deep water [i.e., representing a sea state where each pair of $H_{s}, T_{p}$ (or $T_{z}$ ) represent one storm condition with a duration of, e.g., $3 \mathrm{~h}$ ]. Different parametric models for the joint probability density function (pdf) of $H_{s}$ and $T_{p}$ or $H_{s}$ and $T_{z}$ are given in the literature. Examples are Moan et al. (2005) (hereafter referred to as MGAU05) and Haver (1985) for $H_{s}$ and $T_{p}$, and Bitner-Gregersen and Guedes Soares (2007) (hereafter referred to as BGGS07) and Mathisen and BitnerGregersen (1990) for $H_{s}$ and $T_{z}$. In the present paper, the statistical properties of $R$ are exemplified using the joint pdf of $H_{S}$ and $T_{p}$ fitted by MGAU05 to 29 years of wave data from the Northern North Sea using the joint Haver (1985) model, as well as the joint pdf of $H_{s}$ and $T_{z}$ fitted by BGGS07 to five data sets from the North Atlantic using the joint BitnerGregersen (1988) model. These pdfs are given as

$p\left(H_{S}, T\right)=p\left(T \mid H_{S}\right) p\left(H_{S}\right)$

where $p\left(H_{s}\right)$ is the marginal pdf of $H_{s}$, which for the MGAU05 distribution is given by the following combined lognormal and Weibull distributions

$p\left(H_{S}\right)=\left\{\begin{array}{l}\frac{1}{\sqrt{2 \pi} \kappa H_{s}} \exp \left[-\frac{\left(\ln H_{s}-\theta\right)^{2}}{2 \kappa^{2}}\right], H_{s} \leq 3.25 \mathrm{~m} \\ \beta \frac{H_{s}^{\beta-1}}{\zeta^{\beta}} \exp \left[-\left(\frac{H_{s}}{\zeta}\right)^{\beta}\right], H_{S}>3.25 \mathrm{~m}\end{array}\right.$

Here, $\theta=0.801, \kappa^{2}=0.371$ are the mean value and the variance, respectively, of $\ln H_{S}$ and $\zeta=2.713, \beta=1.531$ are the Weibull parameters.

For the BGGS07 distribution, $p\left(H_{S}\right)$ is given by the following three-parameter Weibull distribution

$p\left(H_{s}\right)=\frac{r}{s}\left(\frac{H_{s}-t}{s}\right)^{r-1} \exp \left[-\left(\frac{H_{s}-t}{s}\right)^{r}\right], H_{s} \geq t$

where $r, s$ and $t$ are the Weibull parameters given in BGGS07, see Table 1 .

$p\left(T \mid H_{s}\right)$ is the conditional pdf of $T$ given $H_{s}$, which for both distributions is given by the lognormal distribution

$p\left(T \mid H_{S}\right)=\frac{1}{\sqrt{2 \pi} \sigma T} \exp \left[-\frac{(\ln T-\mu)^{2}}{2 \sigma^{2}}\right]$

where $\mu$ and $\sigma^{2}$ are the mean value and the variance, respectively, of $\ln T$. For the MGAU05 distribution, $T=T_{p}$ and (Gao 2007) 
Table 2 Mean value of $\ln T_{z}$, see Eq. (11)

\begin{tabular}{llll}
\hline BGGS07 & $a_{1}$ & $a_{2}$ & $a_{3}$ \\
\hline Dataset 1 & 1.350 & 0.366 & 0.392 \\
Dataset 2 & 1.365 & 0.375 & 0.453 \\
Dataset 3 & 0.790 & 0.805 & 0.292 \\
Dataset 4 & 0.835 & 1.139 & 0.119 \\
Dataset 5 & 1.952 & 0.168 & 0.499 \\
\hline
\end{tabular}

Table 3 Standard deviation of $\ln T_{z}$, see Eq. (11)

\begin{tabular}{llll}
\hline BGGS07 & $b_{1}$ & $b_{2}$ & \multicolumn{1}{c}{$b_{3}$} \\
\hline Dataset 1 & 0.020 & 0.165 & -0.166 \\
Dataset 2 & 0.033 & 0.285 & -0.752 \\
Dataset 3 & 0.055 & 0.195 & -0.269 \\
Dataset 4 & 0.140 & 0.030 & -0.958 \\
Dataset 5 & 0.070 & 0.066 & -0.081 \\
\hline
\end{tabular}

$\mu=a_{1}+a_{2} H_{s}^{a_{3}},\left(a_{1}, a_{2}, a_{3}\right)=(1.780,0.288,0.474)$

$\sigma^{2}=b_{1}+b_{2} e^{b_{3} H_{s}},\left(b_{1}, b_{2}, b_{3}\right)=(0.001,0.097,-0.255)$

For the BGGS07 distribution, $T=T_{z}$ and

$\mu=a_{1}+a_{2} H_{s}^{a_{3}}, \sigma=b_{1}+b_{2} H_{s}^{b_{3}}$

where the parameters in $\mu, \sigma$ are given in BGGS07, see Tables 2 and 3. All these data represent wave conditions in the North Atlantic. Data sets 1, 2 and 3 are numerically generated wave data taken from global databases representing 44 years $(1958-2004)$ at $59^{\circ} 00^{\prime} \mathrm{N}, 19^{\circ} 00^{\prime} \mathrm{W}$. Data set 4 refers to Global Wave Statistics (GWS) zone 9 (the zone located south of Iceland and west of UK) representing visual observations collected from ship in normal service all over the world in the period 1949-1986. Data set 5 refers to Juliet shipborne wave recorder (SBWR) representing data registered at the Ocean Weather Station Juliet during 13 years since 1952 at $52^{\circ} 00^{\prime} \mathrm{N}, 20^{\circ} 00^{\prime} \mathrm{W}$. More details are given in Bitner-Gregersen and Guedes Soares (2007).

\section{Statistical properties of runup}

Statistical properties of $R$ (from which the statistical properties of $R_{2}$ can be obtained) can be derived using the joint pdf of $H_{S}$ and $T$, e.g., giving the joint pdf of $R$ and $H_{s}$. This is obtained from Eq. (4) by a change of variables from $\left(H_{S}, T\right)$ to $\left(H_{s}, R\right)$, which takes the form

$p\left(H_{s}, R\right)=p\left(R \mid H_{S}\right) p\left(H_{S}\right)$
It should be noted that this change of variables only affects $p\left(T \mid H_{S}\right)$ since $T=R / \sqrt{H_{S}}$, yielding a lognormal pdf of $R$ given $H_{s}$ in the form (by using the Jacobian $|\partial R / \partial T|=$ $\left.\sqrt{H_{S}}\right)$

$p\left(R \mid H_{s}\right)=\frac{1}{\sqrt{2 \pi} \sigma_{R} R} \exp \left[-\frac{\left(\ln R-\mu_{R}\right)^{2}}{2 \sigma_{R}^{2}}\right]$

where $\mu_{R}$ and $\sigma_{R}^{2}$ are the conditional mean value and the conditional variance, respectively, of $\ln R$, given by

$\mu_{R}=\mu+\frac{1}{2} \ln H_{s} ; \sigma_{R}^{2}=\sigma^{2}$

where $\mu$ and $\sigma^{2}$ are given in Eqs. (9) and (10) for the MGAU05 distribution and in Eq. (11) for the BGGS07 distribution.

The cumulative distribution function (cdf) of $R$ given $H_{S}$ is obtained from

$P\left(R \mid H_{S}\right)=\Phi\left[\frac{\ln R-\mu_{R}}{\sigma_{R}}\right]$

where $\Phi$ is the standard Gaussian cdf given by

$\Phi(v)=\frac{1}{\sqrt{2 \pi}} \int_{-\infty}^{v} e^{-t^{2} / 2} d t$

The expected value of $R$ given $H_{S}$ is given by (Bury 1975)

$E\left[R \mid H_{S}\right]=\exp \left(\mu_{R}+\frac{1}{2} \sigma_{R}^{2}\right)$

The standard deviation of $R$ given $H_{S}$ is given by (Bury 1975)

$\sigma\left[R \mid H_{s}\right]=\left[\left(e^{\sigma_{R}^{2}}-1\right) \exp \left(2 \mu_{R}+\sigma_{R}^{2}\right)\right]^{1 / 2}$

\section{Examples of results}

Here, examples of results are given by adopting the new formulation proposed by de la Pena et al. (2014) given by Eq. (1) by taking

$K=4 m^{0.3}$

and valid for $\xi_{p}<0.6$. Their results are based on physical model experiments with a sand seabed using two grain sizes performed for the estimation of extreme runup for the three beach slopes $m=1 / 50,1 / 30,1 / 20$ [see de la Pena et al. (2014) for more details]. Thus, Eq. (4) takes the form

$R \equiv \frac{R_{2}}{c \cdot 4 m^{1.3} \sqrt{g / 2 \pi}}=T \sqrt{H_{S}}$

Moreover, $c=1$ for $T=T_{p}$ and $c=1.28$ for $T=T_{z}$; where the latter is taken from Myrhaug and Kjeldsen (1987, Fig. 11) where $T_{p}=1.28 T_{z}$ for a JONSWAP spectrum with 
Table 4 Example of results for wave runup on shorelines based on the de la Pena et al. (2014) formulation and the MGAU05 and BGGS07 distribution results for $H_{s}=7.5 \mathrm{~m}$ and slope $m=1 / 20$

\begin{tabular}{lll}
\hline Distribution & $E\left[R_{2} \mid H_{s}=7.5 \mathrm{~m}\right](\mathrm{m})$ & $\sigma\left[R_{2} \mid H=7.5 \mathrm{~m}\right](\mathrm{m})$ \\
\hline MGAU05 & 3.52 & 0.44 \\
BGGS07 & & \\
Dataset 1 & 3.12 & 0.47 \\
Dataset 2 & 3.57 & 0.34 \\
Dataset 3 & 3.40 & 0.58 \\
Dataset 4 & 3.53 & 0.51 \\
Dataset 5 & 4.01 & 0.51 \\
\hline
\end{tabular}

peakedness factor $\gamma=3.3$. However, it should be noted that this relationship between $T_{p}$ and $T_{z}$ is not necessarily valid for the data sets used here. They might contain mixed swell and wind sea for which other relationships exist.

The given flow conditions are (e.g., representing one storm condition with a duration of $3 \mathrm{~h}$ ):

- Significant wave height in deep water, $H_{s}=7.5 \mathrm{~m}$

- Slope of beach, $m=1 / 20$

Since the de la Pena et al. (2014) formulation is valid for $\xi_{p}<0.6$, it is required that $T_{p}<26.3 \mathrm{~s}$ and $T_{z}<20.5 \mathrm{~s}$.

For the runup it follows from Eqs. (20) and (17) that

$E\left[R_{2} \mid H_{S}=7.5 \mathrm{~m}\right]=c 4 m^{1.3} \sqrt{\frac{g}{2 \pi}} E\left[R \mid H_{S}=7.5 \mathrm{~m}\right]$

and from Eqs. (20) and (18) that

$\sigma\left[R_{2} \mid H_{s}=7.5 \mathrm{~m}\right]=c 4 m^{1.3} \sqrt{\frac{g}{2 \pi}} \sigma\left[R \mid H_{s}=7.5 \mathrm{~m}\right]$

The MGAU05 distribution results are obtained by combining Eqs. (21) and (22) for $c=1$ with Eqs. (14), (9) and (10). The BGGS07 distribution results are obtained by combining Eqs. (21) and (22) for $c=1.28$ with Eqs. (14) and (11). The results are presented in Table 4, showing that the mean values of $R_{2}$ are in the range 3-4 m with standard deviations in the range $0.3-0.5 \mathrm{~m}$ depending on the distribution and data set considered. More explicitly this means that, e.g., based on the MGAU05 distribution results, the expected maximum runup is $3.52 \mathrm{~m}$ with a standard deviation of $0.44 \mathrm{~m}$ in a sea state (one storm condition) with $H_{s}=7.5 \mathrm{~m}$ and a duration of, e.g., $3 \mathrm{~h}$. The actual duration of the sea state can only be obtained if duration statistics are available.

\section{Comments}

Finally, some comments are given on the present method versus common practice in coastal engineering. For assess- ment of, e.g., maximum runup height on beaches and coastal structures common practice would be to start from available data on joint statistics of $H_{s}$ and $T_{p}$ (or $T_{z}$ ) within directional sectors at a nearby offshore location; then to transform these using a wave simulation model to obtain joint statistics of $H_{s}$ and $T_{p}$ (or $T_{z}$ ) at the relevant near-shore location; then to use this information as input for the assessment of maximum wave runup height. Alternatively, the present method provides a simple analytical tool giving first estimates of maximum runup on beaches and coastal structures for given values of $H_{s}, T_{p}$ (or $T_{z}$ ). Such estimates are useful for comparison and verification of more complete computational methods, as well as in situations when time and access to computational resources are limited (under, e.g., field conditions). Moreover, it might also serve as a first inexpensive estimate of the quantities of interest before eventually applying more workintensive computational tools. Although the present results are valid for the specifically chosen wave runup formulation and joint distributions of $\left(H_{s}, T_{p}\right)$ and $\left(H_{s}, T_{z}\right)$ it gives an analytically based method which can be used for other wave runup formulations and joint distributions of $H_{s}$ and $T_{p}$ (or $T_{z}$ ).

\section{Summary}

A simple analytical method which can be used to give estimates of the wave runup on shorelines based on statistics of long-term observation of wave conditions is provided. This is achieved by providing bivariate distribution of $H_{S}$ with the wave runup. The wave runup formulation by de la Pena et al. (2014) defined in terms of $H_{s}$ and $T_{p}$ is adopted. This bivariate distribution of $H_{S}$ and wave runup is obtained by transformations of joint distributions of $\left(H_{s}, T_{p}\right)$ and $\left(H_{s}, T_{z}\right)$ based on fitting to data from the Northern North Sea and the North Atlantic, respectively. The wave runup parameters such as the conditional expected values and the conditional variances for given significant wave height as well as examples of results corresponding to typical field conditions are presented. The present analytical method can be used to calculate estimates of wave runup on shorelines based on, for example, global wave statistics.

\section{References}

Bitner-Gregersen EM (1988) Joint environmental model. Annex A. In: Madsen HO, Pooney P, Bitner-Gregersen E (eds) Probabilistic calculation of design criteria for ultimate tether capacity of Snorre TLP. Det Norske Veritas Report No. 87-31, Høvik, Norway

Bitner-Gregersen E, Guedes Soares C (2007) Uncertainty of average steepness prediction from global wave databases. In: Guedes Soares C, Das PK (eds) Advancements in marine structures, Taylor and Francis Group, London, pp 3-10 
Bury KV (1975) Statistical models in applied science. Wiley, New York Dean RG, Dalrymple RA (1984) Water wave mechanics for engineers and scientists. Prentice-Hall Inc, New Jersey

de la Pena JM, Sanchez-Gonzales JF, Diaz-Sanches R (2014) Wave runup in a sand bed physical model. Waterw Port Coast Ocean Eng 140(4):04014015-1-04014015-11

Gao Z (2007) Private communication. Department of Marine Technology, Norwegian University of Science and Technology, Trondheim, Norway

Haver S (1985) Wave climate off Northern Norway. Appl Ocean Res $7(2): 85-92$
Mathisen J, Bitner-Gregersen E (1990) Joint distributions for significant wave height and wave zero-up-crossing period. Appl Ocean Res 12(2):93-103

Moan T, Gao Z, Ayala-Uraga E (2005) Uncertainty of wave-induced response of marine structures due to long-term variation of extratropical wave conditions. Mar Struct 18(4):359-382

Myrhaug D, Kjeldsen SP (1987) Prediction of occurrences of steep and high waves in deep water. J Waterw Port Coast Ocean Eng 113(2):122-138 